\title{
An Integrated Receiver With Phase-Locked Superconducting Oscillator
}

\author{
Sergey V. Shitov, Valery P. Koshelets, Andrey B. Ermakov, Pavel N. Dmitriev, Lyudmila V. Filippenko, \\ Vladimir V. Khodos, Vladimir L. Vaks, Pavel A. Yagoubov, Willem-Jan Vreeling, and Paul R. Wesselius
}

\begin{abstract}
A submillimeter heterodyne spectrometer employing a superconducting local oscillator is demonstrated for the first time. The sensor chip comprises a quasioptical double-dipole lens-antenna SIS mixer $\left(T_{\mathrm{RX}}=250 \mathrm{~K}\right.$ at $\left.380 \mathrm{GHz}\right)$, a Josephson flux-flow oscillator and a SIS harmonic mixer. Room temperature PLL electronics is used with a reference source at $10 \mathrm{GHz}$. The PLL bandwidth of $10 \mathrm{MHz}$ and the hold range of $3 \mathrm{GHz}$ are estimated for locking at 32-th harmonic of the reference source. The spectral resolution better than $1 \mathrm{MHz}$ and broadening effect of a spectral line of $\mathrm{SO}_{2}$ gas at $326867 \mathrm{MHz}$ are measured with a laboratory gas cell at $300 \mathrm{~K}$ at pressure $0.03-0.3$ mbar using acousto-optical spectrometer.
\end{abstract}

Index Terms-Heterodyne spectrometer, Josephson flux-flow oscillator, phase-locked oscillator, quasioptical SIS receiver.

\section{INTRODUCTION}

$\mathbf{S}$ UB-MILLIMETER wave spectrometers are currently of great interest for radio astronomy and for Earth atmosphere study. Most of advanced spectrometers nowadays employ ultra-low noise SIS mixers at the temperature of liquid helium. The sensor of a SIS mixer is a thin-film circuit fabricated with micron accuracy so this technology is naturally applicable for integrated circuits. In contrast, conventional local oscillators used with SIS mixers are room temperature semiconductor devices (usually a Gunn oscillators in combination with multipliers). This approach makes difficult integration of the whole receiving system.

A superconducting Josephson flux-flow oscillator (FFO) is proven to be suitable for integration with a low-noise SIS mixer as a local oscillator (LO). Among Josephson devices the FFO has an advantage of good tuneability in combination with a relatively narrow free-running linewidth. Recent experimental study on FFO demonstrated possibility of phase-locking up to $700 \mathrm{GHz}$ [1]; this is a good basis for development of a practicable submillimeter spectrometer [2]. Present paper deals with

Manuscript received August 5, 2002. This work was supported in part by RFBR Project 00-02-16270, INTAS Project 01-0367, and the Nederlandse Organizatie voor Wetenschappelijk Onderzoek (NWO).

S. V. Shitov, V. P. Koshelets, A. B. Ermakov, P. N. Dmitriev, and L. V. Filippenko are with the Institute of Radio Engineering and Electronics (IREE), Russian Academy of Sciences, 101999 Moscow, Russia (e-mail: sergey@hitech.cplire.ru).

V. V. Khodos and V. L. Vaks are with the Institute for Physics of Microstructures (IPM), Russian Academy of Sciences, Nizhny Novgorod, Russia (e-mail: vax@ipm.sci-nnov.ru).

P. A. Yagoubov, W.-J. Vreeling, and P. R. Wesselius are with the National Institute for Space Research (SRON), Groningen, The Netherlands (e-mail: p.a.yagoubov@sron.rug.nl).

Digital Object Identifier 10.1109/TASC.2003.813998

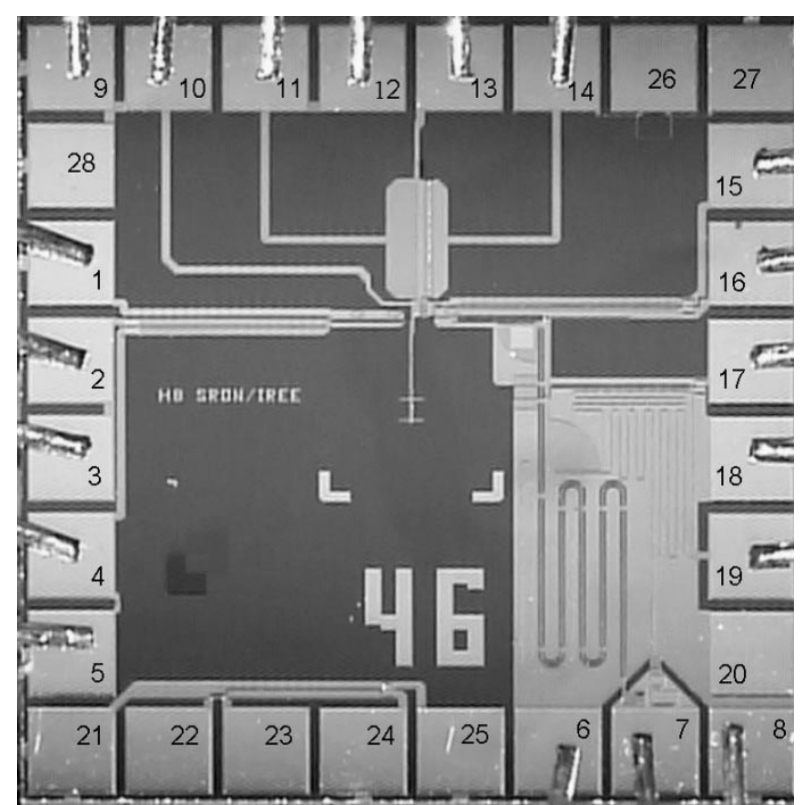

Fig. 1. Chip of superconducting integrated receiver with phase-locked Josephson oscillator $(4 \mathrm{~mm}$ by $4 \mathrm{~mm}$ ). Connections shown: 1,2 contact pads of SIS mixer; 3, 4 SIS control line; 5 bias for balanced mixer (optional); 6-8 reference input and bias for harmonic mixer; 9, 11, 12, 14 FFO bias; 10, 13 FFO control line; 15, 16 harmonic mixer control line; 17, 18 SIS multiplier control line (optional); 19, 20 SIS multiplier bias (optional); 21-27 test structures; 28 spare FFO grounding.

study of a superconducting integrated receiver (SIR) containing the phase-locked FFO as a local oscillator and a quasioptical lens-antenna SIS mixer.

\section{EXPERIMENTAL DETAILS}

\section{A. Chip Design and Measuring System}

The microphotograph of the PLL SIR chip for $320-370 \mathrm{GHz}$ band is presented in Fig. 1. The equivalent scheme of the experimental chip containing the double-dipole single-junction SIS mixer, PLL feedback FFO and harmonic mixer is presented elsewhere [3], [4]. A twin-SIS junction used for the harmonic mixer is operating without magnetic field supply. Fig. 2 presents the general view of the pixel with three coaxial cables mounted. The microwave lens from silicon with antireflection coating is clearly seen. The chip mount is placed inside a magnetic shield as described in [5]. The block scheme of the experimental setup is described elsewhere [6]. We note only that, to reduce length of the PLL loop, the phase detector system is mounted to the wall of the cryostat. 


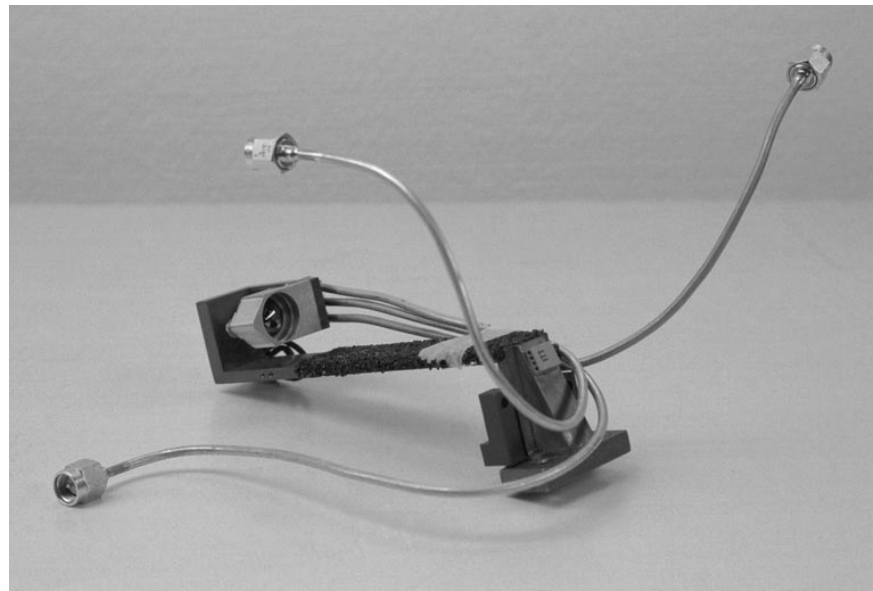

Fig. 2. General view of PLL SIR pixel. The mount of the imaging array is adapted. Three cables are connected to i) SIS mixer for IF/dc-bias, ii) FFO for PLL feedback, iii) harmonic SIS mixer for reference/PLL-IF/dc-bias.

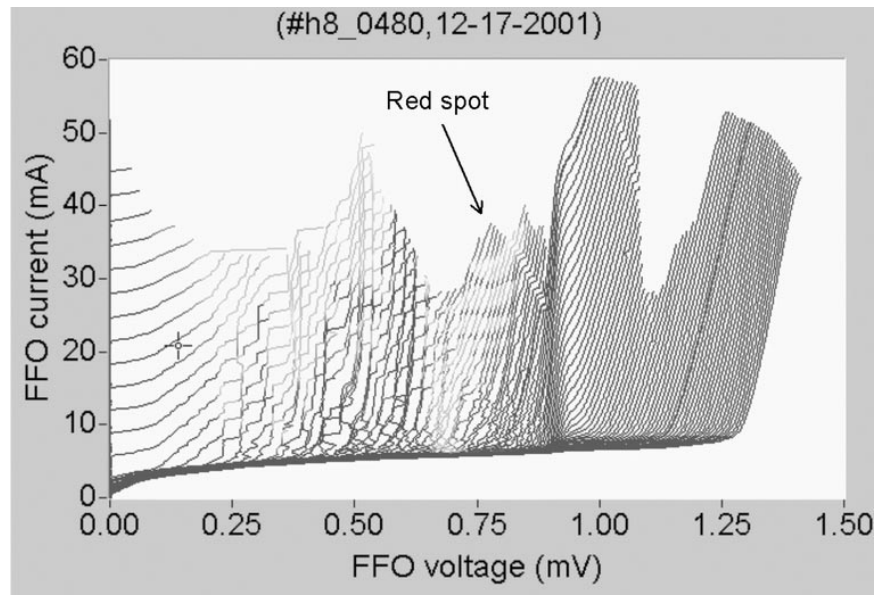

Fig. 3. Test graph of mixer pump on regimes of FFO tuned by magnetic field.

\section{B. Experimental Results and Discussion}

The experimental devices are produced using our standard procedure developed for integrated receivers and described elsewhere [7]. The preliminary test of a device at dc is performed using the computer control measurement system IRTECON [8]. Fig. 3 presents a family of $I V$-curves of FFO for different strength of magnetic field provided by an integrated control line. The data points of each $I V$-curve get different color from blue to red indicating strength of SIS mixer pump. The red 'spot' level corresponds to regimes of FFO suitable to operate the SIS mixer. Similar graph is used for indicating pump level of the harmonic mixer (HM). An important feature of the device is absence of Fiske steps within certain region below the boundary voltage of $V_{g} / 3$ [2]. The permanent tuning is available in this region. This unusual behavior, which would mean normally high damping of the free-running FFO caused by losses, can be explained here by complete disappearance of reflection at the end of the long junction provided by a perfectly matched output circuit. The phase-locking within such region of high damping is known to be difficult due to the wide initial linewidth associated with relatively high dynamic resistance [6].

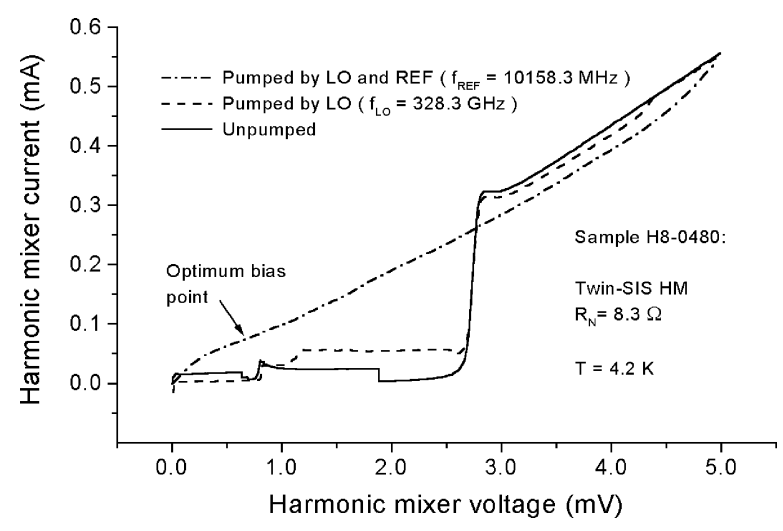

Fig. 4. IV -curves of harmonic mixer pumped with FFO and reference source.

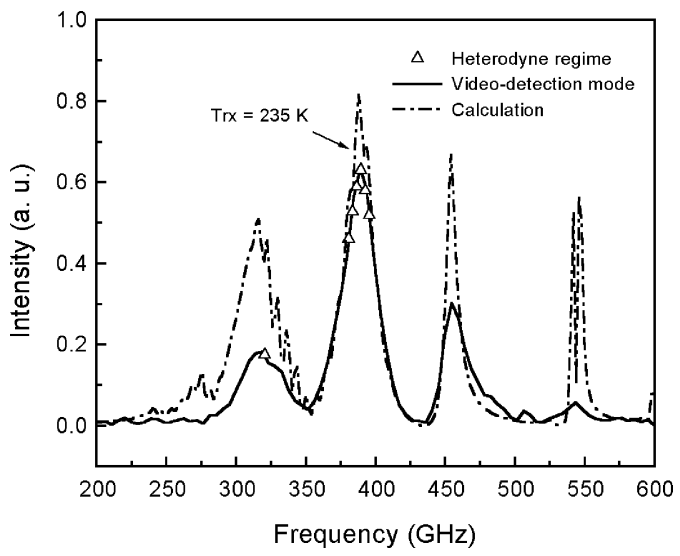

Fig. 5. Experimental FTS and heterodyne data along with best fit of calculated coupling between the antenna and the detector SIS junction.

The $I V$-characteristics of the twin-SIS harmonic mixer (HM) are presented in Fig. 4. To obtain the optimal performance of the HM, it has to be pumped strongly by the reference source at about $10 \mathrm{GHz}$ that turns $I V$-curve of $\mathrm{HM}$ almost into a straight line. The $I V$-characteristic of the detector SIS junction demonstrates no essential influence of the reference source at $10.1 \mathrm{GHz}$ that mean proper rf isolation. The only effects seen, when the reference source is switched on and off, are partial suppression of the critical current (when FFO is off) and negligible change in the quasiparticle current (when the FFO is on).

The Fourier transform spectrum (FTS) of the SIS mixer is obtained using Michelson interferometer as shown in Fig. 5. It was found that the main peak of sensitivity at $380 \mathrm{GHz}$ is essentially narrower and a bit higher in frequency than it was predicted initially by design, $50 \mathrm{GHz}$ and $345 \mathrm{GHz}$ respectively [3], [4]. The result of numerical analysis is also presented in Fig. 5 as the best fit to the experimental data. The fit demonstrates that present results can be explained by thinner insulation $(190 \mathrm{~nm}$ instead of $250 \mathrm{~nm}$ ). The inverse values of experimental heterodyne data $\left(T_{\mathrm{rx}}^{-1}\right)$ are plotted at the same graph demonstrating good fit to the FTS.

The noise temperature of the receiver was measured using the standard hot/cold technique. The heterodyne response of the receiver at $I F=1.4 \mathrm{GHz}$ is presented in Fig. 6 . Note that the peak of highest sensitivity of the detector from Fig. 5 is, unfortunately, within the region of high dynamic resistance, $R_{d}$, 


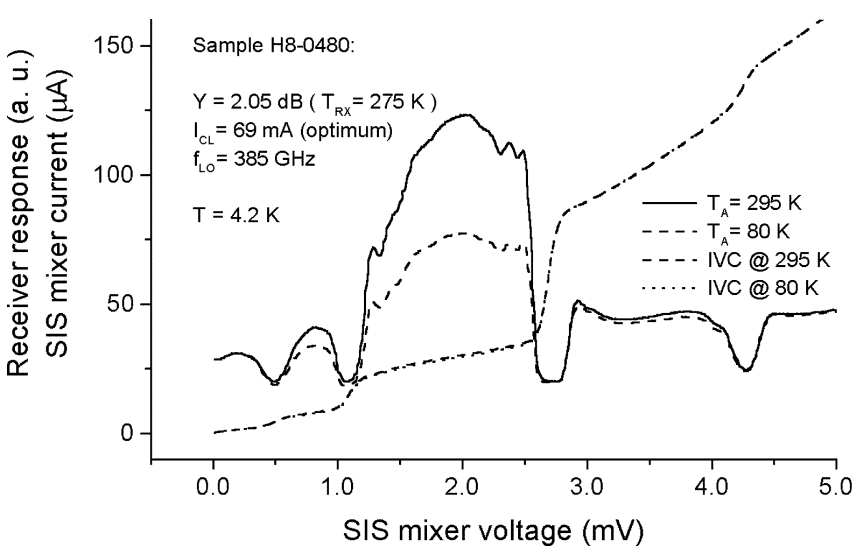

Fig. 6. Receiver heterodyne response at $I F=1.4 \mathrm{GHz}$ and $I V$-curves of SIS mixer pumped by free-running FFO at $385 \mathrm{GHz}$.

of the FFO (see Fig. 3). It is known that for higher $R_{d}$ spectrum of a free-running FFO is affected more by the noise current. In our case the spectrum appeared as a multi-peak fine resonant structure, which is typical for a rectangular-end FFO [9], so the frequency region of the best sensitivity could not be used for the locking of the FFO. For this reason the left (less sensitive) peak of the detector response (at about $320 \mathrm{GHz}$ ) was used for testing the spectrometer.

A spectral line of $\mathrm{SO}_{2}$ gas at $326867.5 \mathrm{MHz}$ was chosen and thus the frequency that FFO has to be tuned to is defined as $325.5 \mathrm{GHz}$. To be sure that the LO is tuned properly, a test with a synthesized $\mathrm{CW}$ signal was performed prior to the gas line detection. The fundamental $\mathrm{CW}$ signal of about $20 \mathrm{GHz}$ was applied to a room temperature Schottky diode mixer to produce a harmonic exactly at the frequency of the spectral line. A beam of the open waveguide of the Schottky mixer was then coupled to the quasioptical input of the receiver.

To phase-lock the FFO at the required frequency of $325.5 \mathrm{GHz}$, the reference PLL signal from main synthesizer was applied at $f_{\mathrm{REF}}=10158.35917 \mathrm{MHz}$. It was assumed that 32-th harmonic of $f_{\mathrm{REF}}$ is separated from the required frequency of FFO by PLL $I F=401 \mathrm{MHz}$ and that the spectral line has to be present at upper sideband (USB) relative to the frequency of the LO. The spectral width of the test $\mathrm{CW}$ signal was found below $10 \mathrm{kHz}$ similar to [6].

The $I V$-curve of FFO phase-locked at $325.5 \mathrm{GHz}$ is presented in Fig. 7. The part of the IVC is magnified in the horizontal direction (see inset) demonstrating loosing and restoring of the locking regime. To obtain these data, the bias current was changed manually within wide range whereas the feedback loop remained closed. The vertical portion of the curve presents the regime of nearly fixed frequency. The real PLL regime remained within the bias current range of 37-43 mA whereas the frequency lock was possible within range of 27.5-43 mA. The PLL regime initial parameters were as following: the control line current $25.2 \mathrm{~mA}$, the bias current $40 \mathrm{~mA}$, the bias voltage of FFO about $673 \mu \mathrm{V}$, the dynamic resistance of the FFO, $R_{d}=$ $0.0015 \Omega$. The hold range of $\pm 2.5 \mathrm{GHz}$ is estimated from the experimental data. The $I V$-curves of the SIS mixer pumped at $325.5 \mathrm{GHz}$ by the phase-locked FFO are presented in Fig. 8. The data are demonstrating possibility of adjustment of LO power

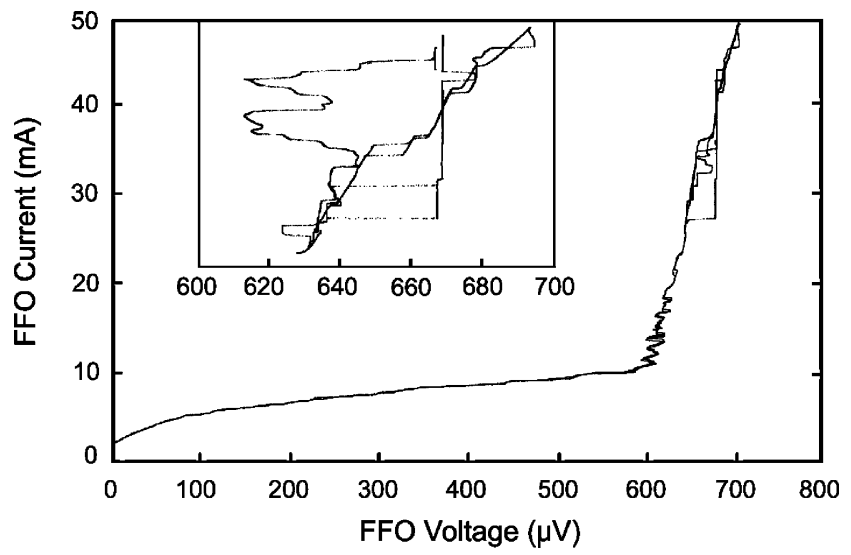

Fig. 7. IV -characteristic of FFO phase-locked at $325.5 \mathrm{GHz}$. The part of the IVC is magnified and presented in the inset demonstrating loosing and restoring of the locking regime for the PLL loop remains closed.

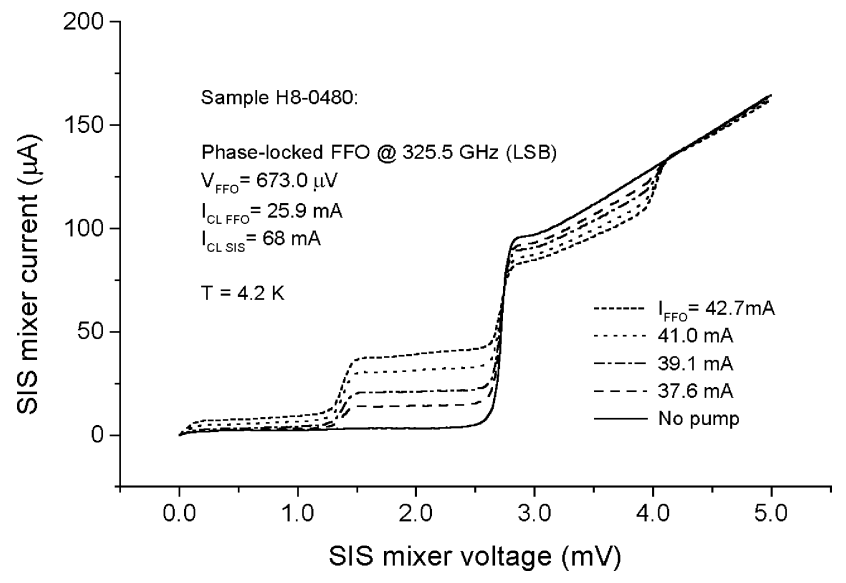

Fig. 8. IV-curves of SIS mixer pumped by phase-locked FFO. The pump level is being adjusted via simply changing the bias current of FFO whereas the superconducting oscillator stays phase-locked.

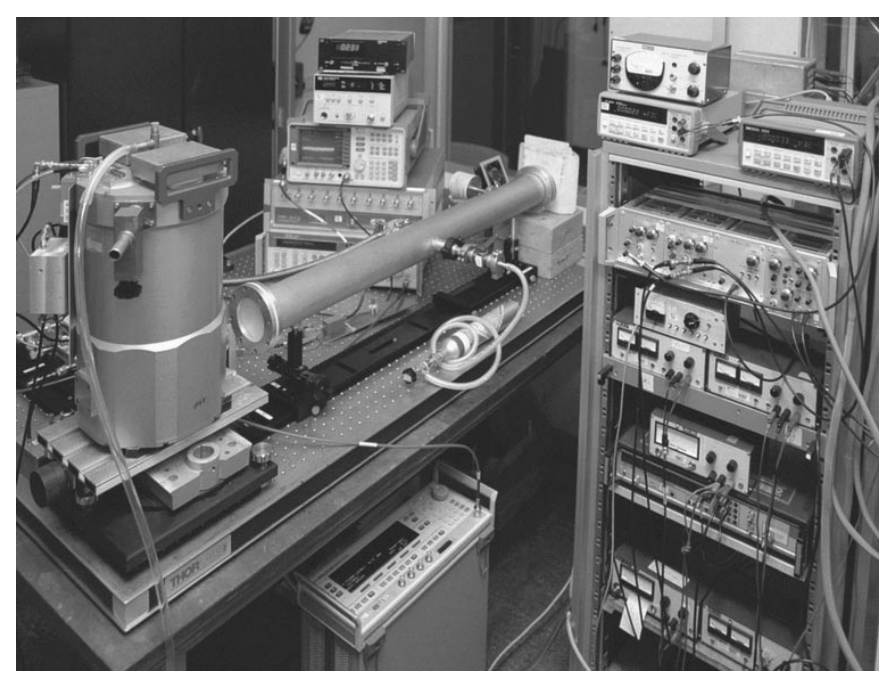

Fig. 9. General view of the gas cell setup.

to its optimum value via changing the bias current whereas the FFO stays phase-locked.

The photograph of our experimental setup for detection of a gas spectral line is presented in Fig. 9. The 1-meter gas cell was 


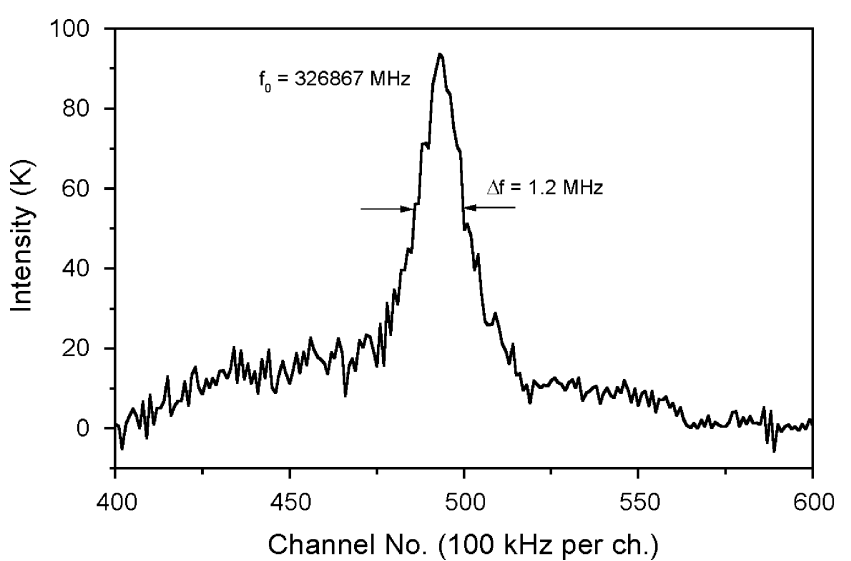

Fig. 10. Spectral line of $\mathrm{SO}_{2}$ gas at pressure 0.03 mbar detected by superconducting integrated receiver with phase-locked Josephson oscillator (FFO). The data obtained using acousto-optical spectrometer.

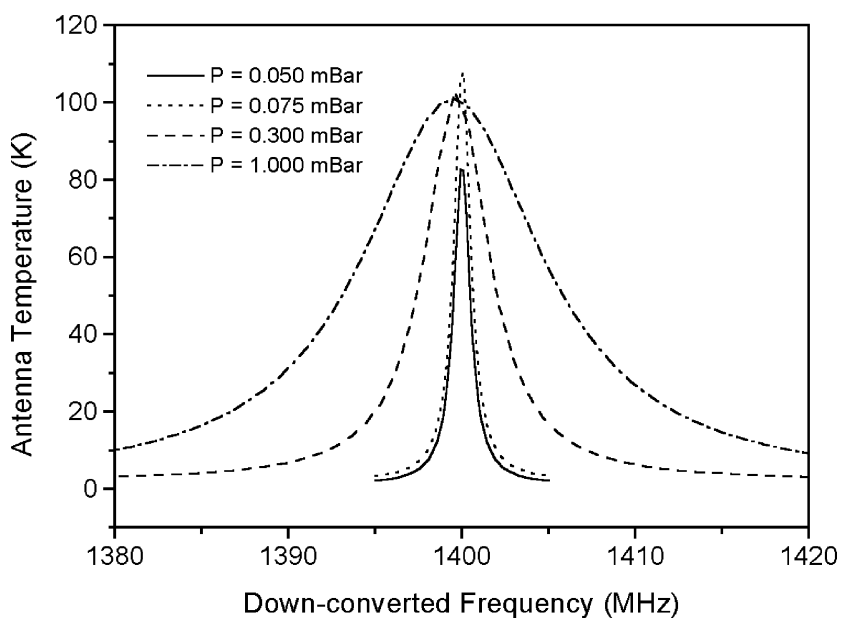

Fig. 11. Effect of broadening of $\mathrm{SO}_{2}$ gas absorption spectrum at $326867 \mathrm{MHz}$ measured by PLL superconducting integrated receiver. The Lorencian fit to experimental data is used.

filled initially with $\mathrm{SO}_{2}$ gas at pressure of a few mbar. Then the cell was pumping down to desired pressure within range of 0.03-1 mbar. The example of a $\mathrm{SO}_{2}$ gas spectrum obtained with acousto-optical spectrometer (AOS) is presented in Fig. 10. The experimental data taken for different pressure were processed using the Lorenz fit similar to [5]. The effect of the linewidth broadening was clearly detected that is presented in Fig. 11.

\section{CONCLUSION}

A sensitive superconducting spectrometer on the base of an integrated externally phase-locked superconducting Josephsontype Flux Flow Oscillator (FFO) is developed for the first time. The spectrometer demonstrated capability of detection of low-pressure atmosphere contaminants like $\mathrm{SO}_{2}$ gas, which is a by-product of the metallurgy industry. The resolution of the spectrometer, as tested with a gas cell using a back-end acousto-optical spectrometer, is better than $1 \mathrm{MHz}$ and can be as good as $10 \mathrm{kHz}$ that is demonstrated with $\mathrm{CW}$ source. To realize full potential of this new technology and reach the quantum noise limit of modern SIS receivers, further improvements of the chip are still necessary both in operation of the FFO and in design of the coupling circuit. This study provides an important input for future development of a balloon-based $500-650 \mathrm{GHz}$ integrated receiver for the TErahertz LImb Sounder (TELIS) scheduled to fly in 2005. The new technology has also good potentials in distant detection of different atmosphere contaminants including Chemical Warfare Agents (CWA).

\section{ACKNOWLEDGMENT}

The authors thank D. Nguen for precision mounting of samples and A. R. W. de Jonge and R. Bernardin for their help with setting up the acousto-optical spectrometer.

\section{REFERENCES}

[1] V. P. Koshelets, P. N. Dmitriev, A. B. Ermakov, A. S. Sobolev, M. Y. Torgashin, V. V. Khodos, V. L. Vaks, P. R. Wesselius, C. Mahaini, and J. Mygind, "Superconducting phase-locked local oscillator for submm integrated receiver," in Proc. 13th Int. Symp. Space Terahertz Technol., Mar. 26-28, 2002.

[2] V. P. Koshelets and S. V. Shitov, "Integrated superconducting receivers," Supercond. Sci. Technol., vol. 13, pp. R53-R69, 2000.

[3] S. V. Shitov, V. P. Koshelets, L. V. Filippenko, P. N. Dmitriev, A. M. Baryshev, W. Luinge, and J.-R. Gao, "Concept of a superconducting integrated receiver with phase-lock loop," in Proc. 10th Int. Symp. Space Terahertz Technol.. Charlottesville, Mar. 1999.

[4] S. V. Shitov, V. P. Koshelets, L. V. Filippenko, P. N. Dmitriev, V. L. Vaks, A. M. Baryshev, W. Luinge, N. D. Whyborn, and J.-R. Gao, "A superconducting integrated receiver with phase-lock loop," in IOP Conf. Ser., vol. 2, 2000, 167, pp. 647-650.

[5] S. V. Shitov, V. P. Koshelets, A. B. Ermakov, L. V. Filippenko, A. M. Baryshev, W. Luinge, and J.-R. Gao, "Superconducting chip receivers for imaging application," IEEE Trans. Appl. Supercond., vol. 9, pp. 3773-3776, 1999.

[6] V. P. Koshelets, S. V. Shitov, P. N. Dmitriev, A. B. Ermakov, L. V. Filippenko, V. V. Khodos, V. L. Vaks, A. M. Baryshev, P. R. Wesselius, and J. Mygind, "Toward a phase-locked superconducting integrated receiver: Prospects and limitations," Physica C, vol. 367, pp. 249-255, 2002.

[7] L. V. Filippenko, S. V. Shitov, P. N. Dmitriev, A. B. Ermakov, V. P. Koshelets, and J. R. Gao, "Integrated superconducting receiver: Fabrication and yield," IEEE Trans. Appl. Supercond., vol. 11, no. 1, pp. 816-819, 2001.

[8] A. B. Ermakov, S. V. Shitov, A. M. Baryshev, V. P. Koshelets, and W. Luinge, "A data acquisition system for test and control of superconducting integrated receivers," IEEE Trans. Appl. Supercond., vol. 11, no. 1 , pp. 840-843, 2001.

[9] V. P. Koshelets, A. B. Ermakov, S. V. Shitov, P. N. Dmitriev, L. V. Filippenko, A. M. Baryshev, W. Luinge, J. Mygind, V. L. Vaks, and D. G. Pavel'ev, "Superfine resonant structure on $I V$-curves of long Josephson junction and its influence on flux flow oscillator linewidth," IEEE Trans. Appl. Supercond., vol. 11, no. 1, pp. 1211-1214, 2001. 